\title{
Saddle-shaped solutions of bistable diffusion equations in all of $\mathbb{R}^{2 m}$
}

Received January 22, 2008 and in revised form June 12, 2008

\begin{abstract}
We study the existence and instability properties of saddle-shaped solutions of the semilinear elliptic equation $-\Delta u=f(u)$ in the whole $\mathbb{R}^{2 m}$, where $f$ is of bistable type. It is known that in dimension $2 m=2$ there exists a saddle-shaped solution. This is a solution which changes sign in $\mathbb{R}^{2}$ and vanishes only on $\left\{\left|x_{1}\right|=\left|x_{2}\right|\right\}$. It is also known that this solution is unstable.

In this article we prove the existence of saddle-shaped solutions in every even dimension, as well as their instability in the case of dimension $2 m=4$. More precisely, our main result establishes that if $2 m=4$, every solution vanishing on the Simons cone $\left\{\left(x^{1}, x^{2}\right) \in \mathbb{R}^{m} \times \mathbb{R}^{m}:\left|x^{1}\right|=\left|x^{2}\right|\right\}$ is unstable outside every compact set and, as a consequence, has infinite Morse index. These results are relevant in connection with a conjecture of De Giorgi extensively studied in recent years and for which the existence of a counter-example in high dimensions is still an open problem.
\end{abstract}

\section{Introduction}

This paper is concerned with the study of bounded solutions of bistable diffusion equations

$$
-\Delta u=f(u) \quad \text { in } \mathbb{R}^{n}
$$

In the last years there has been special interest in a symmetry property of certain solutions. It consists of establishing whether every monotone solution $u$ of (1.1) depends only on one Euclidean variable or, equivalently, whether the level sets of such solutions are all hyperplanes. This question was raised by De Giorgi [13] in 1978, who conjectured that the level sets of every bounded, monotone in one direction, solution of the Allen-Cahn equation

$$
-\Delta u=u-u^{3} \quad \text { in } \mathbb{R}^{n}
$$

must be hyperplanes, at least if $n \leq 8$. The conjecture has been proven to be true for $n=2$ by Ghoussoub and Gui [17], and for $n=3$ by Ambrosio and Cabré [5]. For $4 \leq n \leq 8$ and assuming an additional limiting condition on $u$, it has been established by Savin [22] (see Section 2 for more details).

X. Cabré: ICREA and Universitat Politècnica de Catalunya, Departament de Matemàtica Aplicada I, Diagonal 647, 08028 Barcelona, Spain; e-mail: xavier.cabre@upc.edu

J. Terra: Universitat Politècnica de Catalunya, Departament de Matemàtica Aplicada I, Diagonal 647, 08028 Barcelona, Spain; e-mail: joana.terra@upc.edu 
The existence of a counter-example in higher dimensions remains open. By a result of Jerison and Monneau [19], the existence of a counter-example to the conjecture in $\mathbb{R}^{n+1}$ would be established if one could prove the existence of a bounded, even with respect to each coordinate, global minimizer of $(1.2)$ in $\mathbb{R}^{n}$. By global minimizer we mean an absolute minimizer of the energy with respect to compactly supported perturbations. On the other hand, by a deep result of Savin [22], for $n \leq 7$ every global minimizer is an odd function of only one Euclidean variable. In particular, an even function with respect to each coordinate cannot be a global minimizer in $\mathbb{R}^{n}$ whenever $n \leq 7$.

The crucial remaining question is whether a global minimizer of (1.2), even with respect to each coordinate, exists in higher dimensions. A natural candidate is expected to be found in the class of saddle-shaped solutions, that is, solutions that depend only on two radial variables $s=\left|x^{1}\right|$ and $t=\left|x^{2}\right|$, change sign in $\mathbb{R}^{n}=\mathbb{R}^{2 m}=\left\{\left(x^{1}, x^{2}\right) \in \mathbb{R}^{m} \times \mathbb{R}^{m}\right\}$ and vanish only on the Simons cone $\mathcal{C}=\{s=t\}$. This cone is of importance in the theory of minimal surfaces and its variational properties are related to the conjecture of De Giorgi. Namely, the cone $\mathcal{C} \subset \mathbb{R}^{2 m}$ has zero mean curvature in all even dimensions (except at the singular point 0 ), but it is a minimal cone (minimal in the variational sense) if and only if $2 m \geq 8$ (see Section 2).

Towards the understanding of this open question on global minimizers, we study here saddle-shaped solutions and their stability properties. To be precise in our statements, we first present the definitions to be used throughout the paper.

Equation (1.1) is the Euler-Lagrange equation associated to the energy functional

$$
\mathcal{E}(v, \Omega):=\int_{\Omega}\left\{\frac{1}{2}|\nabla v|^{2}+G(v)\right\} d x, \quad \text { where } \quad G^{\prime}=-f
$$

and $\Omega \subset \mathbb{R}^{n}$ is a bounded domain. The energy $\mathcal{E}$ leads to the following notions of minimality, stability, and Morse index of bounded solutions.

Definition 1.1. Let $f \in C^{1}(\mathbb{R})$.

(a) We say that a bounded $C^{1}$ function $u: \mathbb{R}^{n} \rightarrow \mathbb{R}$ is a global minimizer of 1 if

$$
\mathcal{E}(u, \Omega) \leq \mathcal{E}(u+\xi, \Omega)
$$

for every bounded domain $\Omega$ and every $C^{\infty}$ function $\xi$ with compact support in $\Omega$.

(b) We say that a bounded solution $u$ of (1.1) is stable if the second variation of energy $\delta^{2} \mathcal{E} / \delta^{2} \xi$ with respect to compactly supported perturbations $\xi$ is nonnegative. That is,

$$
Q_{u}(\xi):=\int_{\mathbb{R}^{n}}\left\{|\nabla \xi|^{2}-f^{\prime}(u) \xi^{2}\right\} d x \geq 0 \quad \text { for all } \xi \in C_{c}^{\infty}\left(\mathbb{R}^{n}\right) .
$$

We say that $u$ is unstable if it is not stable.

(c) We say that a bounded solution $u$ of (1.1) has finite Morse index equal to $k \in$ $\{0,1,2, \ldots\}$ if $k$ is the maximal dimension of a subspace $X_{k}$ of $C_{c}^{1}\left(\mathbb{R}^{n}\right)$ such that $Q_{u}(\xi)<0$ for every $\xi \in X_{k} \backslash\{0\}$. Here $C_{c}^{1}\left(\mathbb{R}^{n}\right)$ is the space of $C^{1}\left(\mathbb{R}^{n}\right)$ functions with compact support and $Q_{u}$ is defined in (1.4). If there is no such finite integer $k$, we say that $u$ has infinite Morse index. 
Clearly, every global minimizer is a stable solution. At the same time, every stable solution has finite Morse index equal to 0 . It is also easy to verify that every solution with finite Morse index is stable outside a compact set (see Theorem 1.4 and its proof for more details). In some references, global minimizers are called "local minimizers", where local refers to the fact that the energy is computed in bounded domains.

The following assumption on $G$ :

$$
G \geq 0=G( \pm M) \quad \text { in } \mathbb{R} \quad \text { and } \quad G>0 \quad \text { in }(-M, M)
$$

for some constant $M>0$, guarantees the existence of an increasing solution of (1.1) in dimension 1 , that is, in all of $\mathbb{R}$, taking values in $(-M, M)$; see Lemma 4.3. In addition, such an increasing solution is unique up to translations of the independent variable. Normalizing it to vanish at the origin, we call it $u_{0}$. Thus, we have

$$
\left\{\begin{array}{l}
u_{0}: \mathbb{R} \rightarrow(-M, M), \\
u_{0}(0)=0, \quad \dot{u}_{0}>0, \\
-\ddot{u}_{0}=f\left(u_{0}\right) \quad \text { in } \mathbb{R} .
\end{array}\right.
$$

We will see that $(1.5)$ is related to the bistable character of $f$. Hypothesis 1.5 is satisfied by $f(u)=u-u^{3}$, for which $G(u)=(1 / 4)\left(1-u^{2}\right)^{2}$ and $M=1$. For this nonlinearity, the solution $u_{0}$ can be computed explicitly and it is given by $u_{0}(\tau)=\tanh (\tau / \sqrt{2})$.

Next, note that for every given $b \in \mathbb{R}^{n}$ with $|b|=1$ and $c \in \mathbb{R}$, the function

$$
u_{b, c}(x)=u_{0}(b \cdot x+c) \quad \text { for } x \in \mathbb{R}^{n}
$$

is a bounded solution of 1.1 . These solutions are called 1-D solutions since they depend only on one Euclidean variable. Equivalently, these are the solutions with every level set being a hyperplane. As a consequence of a result of Alberti, Ambrosio, and the first author [2], it is now known that, under hypothesis 1.5 on the nonlinearity, every 1-D solution $u_{b, c}$ is a global minimizer of (1.1). In particular, $u_{b, c}$ is a stable solution.

Furthermore, by a result of Savin [22] in connection with the conjecture of De Giorgi, we now know that 1-D solutions are the only global minimizers of the Allen-Cahn equation $(1.2)$ in $\mathbb{R}^{n}$ for $n \leq 7$. On the other hand, as mentioned before (see Theorem 2.1 in the next section for more details), trying to find a counter-example to the conjecture in higher dimensions (still an open problem) is related to the possibility of finding certain global minimizers in dimensions $n \geq 8$ which are not 1-D. More precisely, the existence of a counter-example to the conjecture in $\mathbb{R}^{n+1}$ would be established if one could prove the existence of a bounded, even with respect to each coordinate, global minimizer of (1.2) in $\mathbb{R}^{n}$. Natural candidates to be minimizers of this type are certain saddle-shaped solutions. The study of their existence and stability properties is the goal of this paper.

The saddle-shaped solutions that we consider are expected to have relevant variational properties due to a well known connection between semilinear equations modeling phase transitions and the theory of minimal surfaces (see Section 2 for details). That connection also motivated De Giorgi to state his conjecture. More precisely, the saddle solutions that we consider are odd with respect to the Simons cone, defined for $n=2 m$ by

$$
\mathcal{C}=\left\{x \in \mathbb{R}^{2 m}: x_{1}^{2}+\cdots+x_{m}^{2}=x_{m+1}^{2}+\cdots+x_{2 m}^{2}\right\} .
$$


It is easy to verify that $\mathcal{C}$ has zero mean curvature at every $x \in \mathcal{C} \backslash\{0\}$, in every dimension $2 m \geq 2$. However, it is only in dimensions $2 m \geq 8$ (besides the case $2 m=2$ ) that this cone is locally stable. In dimensions $2 m \geq 8$ it is in addition a minimizer of the area functional, that is, it is a minimal cone (in the variational sense); see [18].

For $x=\left(x_{1}, \ldots, x_{2 m}\right) \in \mathbb{R}^{2 m}$, we define two radial variables $s$ and $t$ by

$$
s=\sqrt{x_{1}^{2}+\cdots+x_{m}^{2}} \geq 0, \quad t=\sqrt{x_{m+1}^{2}+\cdots+x_{2 m}^{2}} \geq 0 .
$$

The Simons cone is given by $\mathcal{C}=\{s=t\}$.

We now introduce our notion of saddle solution. These solutions depend only on $s$ and $t$, and are odd with respect to $\mathcal{C}$.

Definition 1.2. Let $f \in C^{1}(\mathbb{R})$ be odd. We say that $u: \mathbb{R}^{2 m} \rightarrow \mathbb{R}$ is a saddle-shaped solution (or simply a saddle solution) of

$$
-\Delta u=f(u) \quad \text { in } \mathbb{R}^{2 m}
$$

if $u$ is a bounded solution of (1.10) and, with s and t defined by (1.9),

(a) $u$ depends only on the variables $s$ and $t$, written $u=u(s, t)$;

(b) $u>0$ in $\mathcal{O}=\{s>t\}$;

(c) $u(s, t)=-u(t, s)$ in $\mathbb{R}^{2 m}$.

It follows from (c) that every saddle solution vanishes on the Simons cone $\mathcal{C}=\{s=t\}$. Note also that saddle solutions are even with respect to each coordinate $x_{i}, 1 \leq i \leq 2 \mathrm{~m}$, as in the result of Jerison-Monneau.

By classical elliptic regularity theory, it is well known that for $f \in C^{1}(\mathbb{R})$, every bounded solution of $-\Delta u=f(u)$ in $\mathbb{R}^{n}$ satisfies $u \in C^{2, \alpha}\left(\mathbb{R}^{n}\right)$ for all $0<\alpha<1$, and thus it is a classical solution. In particular, saddle solutions are classical solutions. See the beginning of Section 3 for more details.

Saddle solutions were first studied by Dang, Fife, and Peletier [12] in dimension $n=2$ for $f$ odd, bistable, and with $f(u) / u$ decreasing for $u \in(0,1)$. They proved the existence and uniqueness of a saddle solution in dimension 2 . They also established monotonicity properties and the asymptotic behavior of the saddle solution. Its instability, already indicated in a partial result of [12], was studied in detail by Schatzman [23]. This paper established that the saddle solution is unstable in $\mathbb{R}^{2}$ by studying the linearized operator at the solution in some appropriate function spaces, and by showing that it has a strictly negative eigenvalue corresponding to an eigenfunction having the symmetries of the square. Moreover, in the case of the Allen-Cahn equation (1.2), the linearized operator was shown to have exactly one negative eigenvalue.

The article [1] studies vector-valued saddle solutions in $\mathbb{R}^{2}$. The recent work [4] concerns scalar saddle type solutions in $\mathbb{R}^{2}$ changing sign on more nodal lines than $x_{1}= \pm x_{2}$.

The instability of the saddle solution in dimension 2 (in the sense of Definition 1.1 ) is nowadays a consequence of a more recent result related to the conjecture of De Giorgi. Namely, [17] and [6] established that, for all $f \in C^{1}$, every bounded stable solution of 1.1 in $\mathbb{R}^{2}$ must be a 1-D solution, that is, a solution depending only on one Euclidean 
variable. In particular, in $\mathbb{R}^{2}$ bounded stable solutions cannot be saddle-shaped. These ideas were further used in [24] for the dimension $n=2$.

To state our results on saddle solutions, given a $C^{1}$ nonlinearity $f: \mathbb{R} \rightarrow \mathbb{R}$ and $M>0$, define

$$
G(u)=\int_{u}^{M} f .
$$

We have $G \in C^{2}(\mathbb{R})$ and $G^{\prime}=-f$. In our results we assume some, or all, of the following conditions on $f$, for some $M>0$ and with $G$ defined as above:

$$
\begin{aligned}
& f \text { is odd; } \\
& G \geq 0=G( \pm M) \quad \text { in } \mathbb{R} \quad \text { and } \quad G>0 \quad \text { in }(-M, M) ;
\end{aligned}
$$

$f$ is concave in $(0, M)$.

Condition (1.13) is actually condition $(1.5)$ presented before in connection with the existence of 1-D solutions. Note that if 1.12$)$ and (1.13) hold, then $f(0)=f( \pm M)=0$. On the other hand, if $f$ is odd in $\mathbb{R}$, positive and concave in $(0, M)$, and negative in $(M, \infty)$, then $f$ satisfies (1.12)-(1.14). Hence, the nonlinearities $f$ that we consider are of "balanced bistable type", while the potentials $G$ are of "double-well type". Our three assumptions (1.12)-11.14) are satisfied for the Allen-Cahn (or scalar Ginzburg-Landau) equation

$$
-\Delta u=u-u^{3} .
$$

In this case we have $G(u)=(1 / 4)\left(1-u^{2}\right)^{2}$ and $M=1$. The three hypotheses also hold for the equation

$$
-\Delta u=\sin (\pi u),
$$

for which $G(u)=(1 / \pi)(1+\cos (\pi u))$.

Our first result establishes the existence of a saddle solution in $\mathbb{R}^{2 m}$ and some of its variational properties.

Theorem 1.3. Let $f \in C^{1}(\mathbb{R})$ satisfy $(1.12)$ and $(1.13)$ for some constant $M>0$, where $G$ is defined by (1.11). Then, for every even dimension $2 m \geq 2$, there exists a saddleshaped solution $u$ as in Definition 1.2 of $-\Delta u=f(u)$ in $\mathbb{R}^{2 m}$.

In addition, $u$ satisfies $|u|<M$ in $\mathbb{R}^{2 m}$, as well as the energy estimate

$$
\mathcal{E}\left(u, B_{R}\right)=\int_{B_{R}}\left\{\frac{1}{2}|\nabla u|^{2}+G(u)\right\} d x \leq C R^{2 m-1} \quad \text { for all } R>1,
$$

where $C$ is a constant independent of $R$, and $B_{R}$ denotes the open ball of radius $R$ centered at 0 .

If in addition $f$ satisfies (1.14), then the second variation of energy $Q_{u}(\xi)$ at $u$, as defined in (1.4), is nonnegative for all functions $\xi \in C^{1}\left(\mathbb{R}^{2 m}\right)$ with compact support in $\mathbb{R}^{2 m}$ and vanishing on the Simons cone $\mathcal{C}=\{s=t\}$. 
As a consequence of the last statement in the theorem, the instability of saddle solutions in low dimensions is related to perturbations which do not vanish on the Simons cone, and hence, which change the zero level set of the solution.

We prove the existence of a saddle solution by first constructing a positive solution in $\mathcal{O}=\{s>t\}$ depending only on $s$ and $t$. For this, we use a variational method. We then obtain the saddle solution in the whole space through odd reflection with respect to the cone $\mathcal{C}$.

Further variational and monotonicity properties of saddle solutions, as well as their asymptotic behavior, will be established in a forthcoming article [9] by the same authors.

Note that for functions $u$ depending only on $s$ and $t$, such as saddle solutions, the energy functional 1.3 becomes

$$
\mathcal{E}(u, \Omega)=a_{m} \int_{\Omega} s^{m-1} t^{m-1}\left\{\frac{1}{2}\left(u_{s}^{2}+u_{t}^{2}\right)+G(u)\right\} d s d t,
$$

where $a_{m}$ is a positive constant depending only on $m$ - here we have assumed that $\Omega \subset$ $\mathbb{R}^{2 m}$ is radially symmetric in the first $m$ variables and also on the last $m$ variables, and we have abused notation by identifying $\Omega$ with its projection in the $(s, t)$ variables. In these variables, the semilinear equation $(1.10$ reads

$$
-\left(u_{s s}+u_{t t}\right)-(m-1)\left(\frac{u_{s}}{s}+\frac{u_{t}}{t}\right)=f(u) \quad \text { for } s>0, t>0 .
$$

The following is our main result. In dimension $n=4$, we establish the instability outside every compact set of all bounded solutions (not necessarily depending on $s$ and $t$ only) that vanish on the Simons cone $\mathcal{C}=\{s=t\}$. As a consequence, the Morse index of such solutions is proved to be infinite.

Theorem 1.4. Let $f \in C^{1}(\mathbb{R})$ satisfy (1.12)-(1.14). Then every bounded solution of $-\Delta u=f(u)$ in $\mathbb{R}^{4}$ that vanishes on the Simons cone $\mathcal{C}=\left\{x_{1}^{2}+x_{2}^{2}=x_{3}^{2}+x_{4}^{2}\right\}$ is unstable. Furthermore, every such solution $u$ is unstable outside every compact set. That is, for every compact set $K$ in $\mathbb{R}^{4}$ there exists $\xi \in C^{1}\left(\mathbb{R}^{4}\right)$ with compact support in $\mathbb{R}^{4} \backslash K$ for which $Q_{u}(\xi)<0$, where $Q_{u}$ is defined in (1.4). As a consequence, u has infinite Morse index in the sense of Definition 1.1

In particular, all the previous statements hold true for every saddle-shaped solution as in Definition 1.2 if $2 m=4$.

As mentioned before, the instability of the saddle solution in dimension 2 was already proven by Shatzman [23]. More recently we have established the instability result also in dimension 6-this is to appear in a forthcoming paper [9]. The computations in Section 6 of the present paper, and the more delicate ones in [9], suggest the possibility of saddle solutions being stable in dimensions $2 m \geq 8$. Such a stability result would be a promising hint towards the possible global minimality of saddle solutions in high dimensions, and hence towards a counter-example to the conjecture of De Giorgi. 
A crucial ingredient in the proof of Theorem 1.4 is the following pointwise estimate.

Proposition 1.5. Let $f \in C^{1}(\mathbb{R})$ satisfy (1.12) and $(1.13)$. If $u$ is a bounded solution of $-\Delta u=f(u)$ in $\mathbb{R}^{2 m}$ that vanishes on the Simons cone $\mathcal{C}=\{s=t\}$, then

$$
|u(x)| \leq\left|u_{0}(\operatorname{dist}(x, \mathcal{C}))\right|=\left|u_{0}\left(\frac{s-t}{\sqrt{2}}\right)\right| \quad \text { for all } x \in \mathbb{R}^{2 m},
$$

where $u_{0}$ is defined by (1.6) and $\operatorname{dist}(\cdot, \mathcal{C})$ denotes the distance to the Simons cone.

In addition, the function $u_{0}((s-t) / \sqrt{2})$ is a supersolution of $-\Delta u=f(u)$ in the set $\mathcal{O}=\{s>t\}$.

This proposition is proven in Section 4 using an important gradient bound of Modica [20] for bounded solutions of 1.1$)$. Instead, its last statement $-u_{0}((s-t) / \sqrt{2})$ being a supersolution in $\{s>t\}$, which by the way will not be used in this paper-follows simply from direct computation using (1.17). Since $|s-t| / \sqrt{2}$ is the distance to the Simons cone, this last statement corresponds to the well known fact that the distance function to a hypersurface of zero mean curvature is superharmonic on each side of the hypersurface.

The heuristic idea behind the instability result of Theorem 1.4 is the following. One expects that the saddle solution behaves at infinity as the transition profile $u_{0}$ placed over the cone $\mathcal{C}$, that is, as $u_{0}((s-t) / \sqrt{2})$ in $(1.18)$. One may expect that this, combined with the instability of the Simons cone in dimensions 4 and 6, could lead to the instability of the saddle solution. In this paper we see that this idea works in dimension 4 thanks to the estimate of Proposition 1.5 .

Indeed, the proof of Theorem 1.4 proceeds as follows. We prove that the quadratic form $Q_{u}$ defined by 1.4 with $u$ replaced by the explicit function $u_{0}((s-t) / \sqrt{2})$ is negative when $n=4$ for some test function $\xi$. This will imply-based on estimate (1.18) and the assumptions on $f$ - that $Q_{u}$ is also negative for some test function, where $u$ is any given solution vanishing on $\mathcal{C}$. That is, $u$ is unstable in dimension $n=4$.

Finally, let us comment on results about the Morse index of stationary surfaces, i.e., surfaces of zero mean curvature. The usual proof of the instability of the Simons cone in dimension 4 and 6 (see [18]) also leads to its instability outside every compact set, and hence to the infinite Morse index property. A precise study of the Morse index of stationary surfaces close to the Simons cone is made in [3] through the analysis of intersection numbers.

The paper is organized as follows. In Section 2 we present the precise statement of the conjecture of De Giorgi and its connections with the variational properties of solutions to (1.2) and with minimal cones. We also recall the result of Jerison and Monneau mentioned above. Section 3 is devoted to the proof of Theorem 1.3 on the existence of saddle solution. Section 4 concerns the proof of Proposition 1.5 , an important tool towards the proof of our instability result, Theorem 1.4 which is presented in Section 5. Finally, in Section 6 we present the asymptotic computations used in the proof of Theorem 1.4 carried out in every dimension $2 m \geq 4$. 


\section{A conjecture of De Giorgi, minimal cones, and saddle solutions}

In 1978 De Giorgi [13] raised the following question:

Conjecture (De Giorgi [13]). Let $u \in C^{2}\left(\mathbb{R}^{n}\right)$ be a solution of

$$
-\Delta u=u-u^{3} \quad \text { in } \mathbb{R}^{n}
$$

such that

$$
|u| \leq 1 \quad \text { and } \quad \partial_{x_{n}} u>0
$$

in the whole $\mathbb{R}^{n}$. Is it true that all level sets $\{u=\lambda\}$ of $u$ are hyperplanes, at least if $n \leq 8$ ?

This conjecture was proved for $n=2$ by Ghoussoub and Gui [17], and for $n=3$ by Ambrosio and Cabré [5]. For $n \leq 8$, a weaker version of the conjecture was proven recently by Savin [22]. Namely, if one further assumes that

$$
\lim _{x_{n} \rightarrow \pm \infty} u\left(x^{\prime}, x_{n}\right)= \pm 1 \quad \text { for all } x^{\prime} \in \mathbb{R}^{n-1}
$$

and $n \leq 8$, then all level sets of $u$ are hyperplanes. We emphasize that, in this result, the limits above are not assumed to be uniform in $x^{\prime} \in \mathbb{R}^{n-1}$.

A related and deep result of Savin [22] is the following. If $u$ is a global minimizer of (1.2) (a local minimizer in the terminology of [22]) and $n \leq 7$, then the level sets of $u$ are hyperplanes. One expects that $n \leq 7$ is optimal in this result. However, the existence for $n \geq 8$ of a global minimizer not being 1-D is still an open problem. In this direction, saddle solutions are natural candidates for being global minimizers (and not 1-D) in high dimensions. More precisely, if their minimality held true in some dimension, this would provide a counter-example to the conjecture of De Giorgi in one more dimension.

Indeed, the connection between the existence of certain global minimizers and the veracity of the conjecture of De Giorgi is established by Jerison and Monneau in [19]. Namely, they prove that if there existed a bounded, even with respect to each coordinate, global minimizer in $\mathbb{R}^{n-1}$, then there would be a bounded solution $u$ to 1.2 , increasing in $x_{n}$ and with one level set not being a hyperplane. That is, this would provide a counterexample to the conjecture in $\mathbb{R}^{n}$. Their precise result is the following.

Theorem 2.1 (Jerison-Monneau [19]). Let $G$ satisfy (1.13) with $M=1$ and assume that there exists a global minimizer $v$ in $\mathbb{R}^{n-1}$ such that $|v|<1$ and $v$ is even with respect to each coordinate $x_{i}, i=1, \ldots, n-1$. Then, for each $\gamma \in(0, \sqrt{2 G(v(0))})$, there exists a solution $u \in C^{2}\left(\mathbb{R}^{n}\right)$ of $\Delta u=G^{\prime}(u)$ in $\mathbb{R}^{n}$ satisfying $|u| \leq 1$ and $\partial_{x_{n}} u>0$ in $\mathbb{R}^{n}$, and such that, for some $\lambda \in \mathbb{R}$, the set $\{u=\lambda\}$ is not a hyperplane.

Moreover, this solution $u$ is a global minimizer in $\mathbb{R}^{n}$, it is even in the first $n-1$ coordinates, and satisfies $\partial_{x_{n}} u(0)=\gamma$ and $u(0)=v(0)$.

It is not known, however, if the solution $u$ of the previous theorem would satisfy $\lim _{x_{n} \rightarrow \pm \infty} u\left(x^{\prime}, x_{n}\right)= \pm 1$ for all $x^{\prime} \in \mathbb{R}^{n}$ as in hypothesis (2.1).

Note that saddle solutions (as in Definition 1.2) are even with respect to each coordinate $x_{i}, 1 \leq i \leq 2 m$, as in the previous theorem (here we would have $n-1=2 m$ ). 
As a first step towards understanding global minimizers with the properties of $v$ in Theorem 2.1. one may study stable solutions-stability being a necessary condition for global minimality. Classifying all bounded stable solutions to (1.1) is a difficult task. A complete characterization of stable solutions is only available in dimension $n=2$. The results of [17] imply that, for all $f$, a nonconstant bounded solution to $\left[1.1\right.$ in $\mathbb{R}^{2}$ is stable if and only if it is 1-D and monotone. The proof of this stability result involves a Liouville-type theorem due to Berestycki, Caffarelli, and Nirenberg [6]. As an immediate corollary of the previous result, we find that, for $n=2$, if $u$ is radially symmetric then it is unstable, since all stable solutions must be 1-D. In higher dimensions, Cabré and Capella [8] have established that, for $n \leq 8$ and all $f \in C^{1}(\mathbb{R})$, if $u$ is a nonconstant bounded radial solution of (1.1), then $u$ is unstable. The same result holds for $9 \leq n \leq 10$ by a more recent result of Villegas [26]. On the other hand, for $n \geq 11$, [8] provides a polynomial $f$ which admits a stable nonconstant bounded radial solution $u$ of $(1.1$. Recent works of Dancer and Farina [14, 15, 11] establish interesting classification results for stable and finite Morse index solutions (general solutions, not only radial, and even unbounded) of supercritical elliptic problems.

The level sets of 1-D solutions and of radial solutions are, respectively, hyperplanes and spheres. Instead, saddle solutions have the Simons cone as a level set, and thus their geometry is more involved. In the remainder of this section we explain the results on minimal graphs and minimal cones that are relevant to the conjecture of De Giorgi and to the variational properties of saddle solutions.

Let $u$ be a bounded solution of $(1.2)$ in all of $\mathbb{R}^{n}$ and consider the blow-down family of functions $\left\{u_{\varepsilon}\right\}$ defined by $u_{\varepsilon}(x)=u(x / \varepsilon)$ for small $\varepsilon$. This is a solution of the same equation with $f$ replaced by $\varepsilon^{-2} f$. The study of the behavior of $u_{\varepsilon}$ as $\varepsilon \rightarrow 0$ leads to some information on $u$ at infinity. It was proven by Modica and Mortola [21] that the energy functionals $\mathcal{E}_{\varepsilon}$ corresponding to $u_{\varepsilon}$ (see [2] for details) $\Gamma$-converge to a multiple of the perimeter functional $\mathcal{P}$ as $\varepsilon \rightarrow 0$. Let us explain this by giving one of its consequences. If $\left\{u_{\varepsilon}\right\}$ is a sequence of minimizers of $\mathcal{E}_{\varepsilon}$, then a subsequence of $u_{\varepsilon}$ converges to a characteristic function $\chi_{E}-\chi_{\Omega \backslash E}$ as $\varepsilon \rightarrow 0$ for which $\partial E \cap \Omega$ is a minimal hypersurface (minimal in the variational sense).

Since the level sets $\left\{u_{\varepsilon}=\lambda\right\}$ are rescaled versions of the level sets $\{u=\lambda\}$ of $u$, the result of Modica and Mortola indicates that the level sets $\{u=\lambda\}$ of $u$ converge at infinity, in some weak sense and along subsequences, to a minimal surface. The minimality of $u$, under the hypothesis of the conjecture and [2.1], is guaranteed by a result of [2]. Since $u$ satisfies the monotonicity condition

$$
\partial_{x_{n}} u>0 \quad \text { in } \mathbb{R}^{n}
$$

each level set of $u$ is the graph of a function from $\mathbb{R}^{n-1}$ to $\mathbb{R}$ along the $x_{n}$ direction. Therefore, the limiting minimal surface should be the graph of a function from $\mathbb{R}^{n-1}$ to $\mathbb{R}$.

The problem of classifying all entire minimal graphs was settled by Simons in [25]. His result establishes that every entire minimal graph of a function from $\mathbb{R}^{k}$ to $\mathbb{R}$ is necessarily a hyperplane for $k \leq 7$. Going back to our problem, we expect that the limiting minimal graph is a hyperplane (that is, the level sets of $u$ are in some sense flat at infinity) 
whenever $k=n-1 \leq 7$, i.e., when $n \leq 8$. The conjecture of De Giorgi raises the question of whether each level set itself is a hyperplane, and not only their limit at infinity and along subsequences.

To prove Simon's result on minimal graphs, one first studies minimal cones; see [18]. Simons [25] proved that all minimal cones of dimension less than or equal to 6 in $\mathbb{R}^{n}$ for $n \leq 7$ are hyperplanes. In addition, he established the existence of a singular cone of dimension $2 m-1$ in $\mathbb{R}^{2 m}$ with zero mean curvature (except at its vertex) and locally stable for the area functional if $2 m \geq 8$. This cone, known as the Simons cone, is defined by

$$
\mathcal{C}=\left\{x \in \mathbb{R}^{2 m}: x_{1}^{2}+\cdots+x_{m}^{2}=x_{m+1}^{2}+\cdots+x_{2 m}^{2}\right\} .
$$

One year later, Bombieri, De Giorgi, and Giusti [7] proved that this cone is not only locally stable but actually a minimal cone, that is, a minimizer of the area functional when $2 m \geq 8$. Moreover, they proved that there exists a minimal graph of a smooth function from $\mathbb{R}^{k}$ to $\mathbb{R}$ which is not a hyperplane when $k \geq 8$.

By our definition, the zero level set of a saddle solution coincides with the Simons cone. Hence we expect the minimality properties of $\mathcal{C}$ in high dimensions to play an important role in the variational properties of saddle solutions.

\section{Existence of saddle solution in $\mathbb{R}^{2 m}$}

In this section we prove the existence of a saddle solution in every even dimension. Before this, let us recall some well known facts about the regularity of weak solutions.

Every bounded solution of $-\Delta u=f(u)$ in $\mathbb{R}^{n}$, with $f \in C^{1}$, satisfies $u \in C^{2, \alpha}\left(\mathbb{R}^{n}\right)$ for all $0<\alpha<1$. In addition, $|\nabla u| \in L^{\infty}\left(\mathbb{R}^{n}\right)$. Indeed, we can apply interior $W^{2, p}$ estimates, with $p>n$, to the equation in every ball $B_{2}(x)$ of radius 2 in $\mathbb{R}^{n}$. We find that

$$
\|u\|_{C^{1}\left(\bar{B}_{1}(x)\right)} \leq C\|u\|_{W^{2, p}\left(B_{1}(x)\right)} \leq C\left\{\|u\|_{L^{\infty}\left(B_{2}(x)\right)}+\|f(u)\|_{L^{p}\left(B_{2}(x)\right)}\right\} \leq C
$$

for some constant $C$ independent of $x \in \mathbb{R}^{n}$. Next, we apply $W^{2, p}$ interior estimates to the equations $-\Delta \partial_{j} u=f^{\prime}(u) \partial_{j} u$, to obtain $W^{3, p}$ and hence $C^{2, \alpha}$ estimates for $u$.

To prove the existence of a saddle solution in $\mathbb{R}^{2 m}=\left\{x=\left(x^{1}, x^{2}\right) \in \mathbb{R}^{m} \times \mathbb{R}^{m}\right\}$, we consider the open set

$$
\mathcal{O}:=\{s>t\}=\left\{\left|x^{1}\right|>\left|x^{2}\right|\right\} \subset \mathbb{R}^{2 m}
$$

note that

$$
\partial \mathcal{O}=\mathcal{C}
$$

Using a variational technique we will construct a solution $u$ in $\mathcal{O}$ satisfying $u>0$ in $\mathcal{O}$ and $u=0$ on $\mathcal{C}=\partial \mathcal{O}$. Then, since $f$ is odd, by odd reflection with respect to the cone $\mathcal{C}$ we obtain a saddle solution in the whole space.

Let $B_{R}$ be the open ball in $\mathbb{R}^{2 m}$ centered at the origin and of radius $R$. In the proof we will consider the open bounded set

$$
\mathcal{O}_{R}:=\mathcal{O} \cap B_{R}=\left\{s>t \text { and }|x|^{2}=s^{2}+t^{2}<R^{2}\right\} .
$$

Note that

$$
\partial \mathcal{O}_{R}=\left(\mathcal{C} \cap \bar{B}_{R}\right) \cup\left(\partial B_{R} \cap \mathcal{O}\right) .
$$


Even though we will not need it in the proof, let us point out that the sets $\mathcal{O}_{R}$ and $\mathcal{O}$ are domains (i.e., open connected sets) in dimensions $2 m \geq 4$ (but clearly not in dimension 2). Indeed, to see that $\mathcal{O}_{R}$ is connected, let $x=\left(x^{1}, x^{2}\right) \in \mathcal{O}_{R}$. We can arc-connect $x$ within $\mathcal{O}_{R}$ to the point $\left(x^{1}, 0\right)$, simply using the path $\left(x^{1}, \sigma x^{2}\right), 0 \leq \sigma \leq 1$. Finally, since $m \geq 2$, the point $\left(x^{1}, 0\right)$ can be arc-connected within $\left\{p \in \mathbb{R}^{m}: 0<|p|<R\right\} \times\{0\}$ to the point $(R / 2,0, \ldots, 0)$. Thus, $\mathcal{O}_{R}$ is arc-connected. It follows that $\mathcal{O}$ is also arc-connected.

Proof of Theorem 1.3. With $\mathcal{O}_{R}$ defined as above, consider the space

$$
\tilde{H}_{0}^{1}\left(\mathcal{O}_{R}\right)=\left\{v \in H_{0}^{1}\left(\mathcal{O}_{R}\right): v=v(s, t) \text { a.e. }\right\}
$$

of $H_{0}^{1}$ functions in the bounded open set $\mathcal{O}_{R}$ which depend only on $s$ and $t$. Equivalently, these are the $H_{0}^{1}\left(\mathcal{O}_{R}\right)$ functions which are invariant under orthogonal transformations in the first $m$ variables and also under orthogonal transformations in the second $m$ variables. Thus, $\tilde{H}_{0}^{1}\left(\mathcal{O}_{R}\right)$ is a weakly closed subspace of $H_{0}^{1}\left(\mathcal{O}_{R}\right)$.

Consider the energy functional in $\mathcal{O}_{R}$,

$$
\mathcal{E}\left(v, \mathcal{O}_{R}\right)=\int_{\mathcal{O}_{R}}\left\{\frac{1}{2}|\nabla v|^{2}+G(v)\right\} d x \quad \text { for } v \in \tilde{H}_{0}^{1}\left(\mathcal{O}_{R}\right) .
$$

Next we show the existence of a minimizer of the functional among functions in this space. Recall that we assume condition 1.13 on $G$, that is,

$$
G \geq 0=G( \pm M) \quad \text { in } \mathbb{R} \quad \text { and } \quad G>0 \quad \text { in }(-M, M) .
$$

Since $\mathcal{E}$ is nonnegative, we can take a minimizing sequence $\left\{u_{R}^{k}\right\}, k=1,2, \ldots$, of $\mathcal{E}$ in $\tilde{H}_{0}^{1}\left(\mathcal{O}_{R}\right)$. Without loss of generality we may assume that $0 \leq u_{R}^{k} \leq M$. To see this, simply replace the minimizing sequence $\left\{u_{R}^{k}\right\}$ by the sequence $\left\{v_{R}^{k}\right\}$ defined by $v_{R}^{k}=\min \left\{\left|u_{R}^{k}\right|, M\right\} \in \tilde{H}_{0}^{1}\left(\mathcal{O}_{R}\right)$, which is also a minimizing sequence. Indeed, $\left\{\left|u_{R}^{k}\right|\right\}$ is a minimizing sequence since $G$ is even; then use the fact that $G \geq G(M)$ to conclude that $\left\{v_{R}^{k}\right\}$ is also minimizing.

Since $G \geq 0$, we have

$$
\int_{\mathcal{O}_{R}}\left|\nabla u_{R}^{k}\right|^{2} d x \leq 2 \mathcal{E}\left(u_{R}^{k}, \mathcal{O}_{R}\right) \leq C
$$

for some constant $C$, and thus there exists a subsequence (denoted again by $\left\{u_{R}^{k}\right\}$ ) such that $u_{R}^{k}$ converges weakly in $H_{0}^{1}\left(\mathcal{O}_{R}\right)$ to a function $u_{R} \in \tilde{H}_{0}^{1}\left(\mathcal{O}_{R}\right)$. Due to the weak convergence we deduce

$$
\int_{\mathcal{O}_{R}}\left|\nabla u_{R}\right|^{2} d x \leq \liminf _{k} \int_{\mathcal{O}_{R}}\left|\nabla u_{R}^{k}\right|^{2} d x .
$$

By Fatou's lemma, we also have

$$
\int_{\mathcal{O}_{R}} G\left(u_{R}\right) d x \leq \liminf _{k} \int_{\mathcal{O}_{R}} G\left(u_{R}^{k}\right) d x .
$$

Hence, $u_{R}$ is a minimizing function in $\tilde{H}_{0}^{1}\left(\mathcal{O}_{R}\right)$ and $0 \leq u_{R} \leq M$ in $\mathcal{O}_{R}$. 
Next, we can consider perturbations $u_{R}+\xi$ of $u_{R}$, with $\xi$ depending only on $s$ and $t$, and with $\xi$ having compact support in $\mathcal{O}_{R} \cap\{t>0\}=B_{R} \cap\{0<t<s\}$. In particular, $\xi$ vanishes in a neighborhood of $\{t=0\}$. Since equation 1.17$)$ in the $(s, t)$ variables is the first variation of $\mathcal{E}\left(\cdot, \mathcal{O}_{R}\right)$-recall that $\mathcal{E}$ has the form 1.16$)$ on $\tilde{H}_{0}^{1}$ functions - and the equation is not singular away from $\{s=0\}$ and $\{t=0\}$, we deduce that $u_{R}$ is a solution of 1.17$)$ in $\mathcal{O}_{R} \cap\{t>0\}$. That is, we have

$$
-\Delta u_{R}=f\left(u_{R}\right) \quad \text { in } \mathcal{O}_{R} \cap\{t>0\} .
$$

We now prove that $u_{R}$ is also a solution in all of $\mathcal{O}_{R}$, that is, also across $\{t=0\}$. To see this for dimensions $2 m \geq 4$, let $\xi_{\varepsilon}$ be a smooth function of $t$ alone, identically 0 in $\{t<\varepsilon / 2\}$ and identically 1 in $\{t>\varepsilon\}$. Let $v \in C_{c}^{\infty}\left(\mathcal{O}_{R}\right)$, multiply (3.2) by $v \xi_{\varepsilon}$ and integrate by parts to obtain

$$
\int_{\mathcal{O}_{R}} \xi_{\varepsilon} \nabla u_{R} \nabla v d x+\int_{\mathcal{O}_{R} \cap\{t<\varepsilon\}} v \nabla u_{R} \nabla \xi_{\varepsilon} d x=\int_{\mathcal{O}_{R}} f\left(u_{R}\right) v \xi_{\varepsilon} d x .
$$

We conclude by seeing that the second integral on the left hand side goes to zero as $\varepsilon \rightarrow 0$. Indeed, by the Cauchy-Schwarz inequality,

$$
\left|\int_{\mathcal{O}_{R} \cap\{t<\varepsilon\}} v \nabla u_{R} \nabla \xi_{\varepsilon} d x\right|^{2} \leq C \int_{\mathcal{O}_{R} \cap\{t<\varepsilon\}}\left|\nabla u_{R}\right|^{2} d x \int_{\mathcal{O}_{R} \cap\{t<\varepsilon\}}\left|\nabla \xi_{\varepsilon}\right|^{2} d x .
$$

Since $\left|\nabla \xi_{\varepsilon}\right|^{2} \leq C / \varepsilon^{2},\left|\mathcal{O}_{R} \cap\{t<\varepsilon\}\right| \leq C_{R} \varepsilon^{m}$, and $m \geq 2$, the second factor in the previous bound is bounded independently of $\varepsilon$. At the same time, the first factor tends to zero as $\varepsilon \rightarrow 0$ since $\left|\nabla u_{R}\right|^{2}$ is integrable in $\mathcal{O}_{R}$.

In dimension $2 m=2$ the previous proof does not apply and we argue as follows. We now consider perturbations $\xi \in \tilde{H}_{0}^{1}\left(\mathcal{O}_{R}\right)$ which do not vanish on $B_{R} \cap\{t=0\}$. Considering the first variation of energy and integrating by parts, we find that the boundary flux $s^{m-1} t^{m-1} \partial_{t} u_{R}=\partial_{t} u_{R}$ (here $m-1=0$ ) must be identically 0 on $B_{R} \cap\{t=0\}$. This implies that $u_{R}$ is a solution also across $\{t=0\}$.

We have established the existence of a solution $u_{R}$ in $\mathcal{O}_{R}=B_{R} \cap\{s>t\}$ with $0 \leq u_{R} \leq M$. Considering the odd reflection of $u_{R}$ with respect to the Simons cone $\mathcal{C}$,

$$
u_{R}(s, t)=-u_{R}(t, s)
$$

we obtain a solution in $B_{R} \backslash\{0\}$. Using the same cut-off argument as above, but choosing now $1-\xi_{\varepsilon}$ to have support in the ball of radius $\varepsilon$ around 0 , we conclude that $u_{R}$ is also a solution around 0 , and hence in all of $B_{R}$. Here, the cut-off argument also applies in dimension 2.

We now wish to pass to the limit in $R$ and obtain a solution in all of $\mathbb{R}^{2 m}$. For this, let $S>0$ and consider the family $\left\{u_{R}\right\}$, for $R>S+2$, of solutions in $B_{S+2}$. Since $\left|u_{R}\right| \leq M$, interior elliptic estimates applied in balls of radius 2 centered at points in $\bar{B}_{S}$ (as explained at the beginning of this section) give a $C^{2, \alpha}\left(\bar{B}_{S}\right)$ bound for $u_{R}$ (uniform with respect to $R$ ). For later purposes, using the argument in 3.1) for $u_{R}$, we have

$$
\left|\nabla u_{R}\right| \leq C \quad \text { in } B_{S} \quad \text { for all } R>S+2,
$$


for some constant $C$ independent of $S$ and $R$. In addition, by the Arzelà-Ascoli theorem, a subsequence of $\left\{u_{R}\right\}$ converges in $C^{2}\left(\bar{B}_{S}\right)$ to a solution in $B_{S}$. Taking $S=1,2,3, \ldots$, by a Cantor diagonal argument, we obtain a sequence $u_{R_{j}}$ converging in $C_{\text {loc }}^{2}\left(\mathbb{R}^{2 m}\right)$ to a solution $u \in C^{2}\left(\mathbb{R}^{2 m}\right)$.

By construction, $u$ is a solution in $\mathbb{R}^{2 m}$ depending only on $s$ and $t$, odd with respect to the Simons cone $\mathcal{C}$, with $|u| \leq M$ in $\mathbb{R}^{2 m}$, and with $u \geq 0$ in $\{s>t\}$. Now, as $f(M)=0$ and $u \neq \equiv M$ (since $u$ vanishes on $\mathcal{C}$ ), the strong maximum principle shows that $u<M$ everywhere. As a consequence, we also have $u>-M$.

We claim that $u \neq \equiv 0$ in $\mathbb{R}^{2 m}$. Then the strong maximum principle leads to $u>0$ in $\{s>t\}$, since $f(0)=0$ and $u \geq 0$ in $\{s>t\}$. Thus, $u$ has all the properties of a saddle solution as in Definition 1.2

To show that $u \neq \equiv 0$, let $1<S<R-2$ and $w_{R}$ be defined as

$$
w_{R}=\xi \min \left\{M, \frac{s-t}{\sqrt{2}}\right\}+(1-\xi) u_{R},
$$

where $\xi$ is a smooth function depending only on $r^{2}=s^{2}+t^{2}$ such that $\xi \equiv 1$ in $B_{S-1}$ and $\xi \equiv 0$ outside $B_{S}$. We see that $w_{R} \in \tilde{H}_{0}^{1}\left(\mathcal{O}_{R}\right)$ satisfies

$$
w_{R}= \begin{cases}u_{R} & \text { in } \mathcal{O}_{R} \backslash \mathcal{O}_{S} \\ \min \left\{M, \frac{s-t}{\sqrt{2}}\right\} & \text { in } \mathcal{O}_{S-1}\end{cases}
$$

In addition, by (3.4), we have

$$
\left|\nabla w_{R}\right| \leq C \quad \text { in } \mathcal{O}_{S}
$$

for some constant $C$ independent of $S$ and $R$.

Since $u_{R}$ minimizes the energy in $\tilde{H}_{0}^{1}\left(\mathcal{O}_{R}\right)$, we have $\mathcal{E}\left(u_{R}, \mathcal{O}_{R}\right) \leq \mathcal{E}\left(w_{R}, \mathcal{O}_{R}\right)$. Now, since $w_{R}=u_{R}$ in $\mathcal{O}_{R} \backslash \mathcal{O}_{S}$, we must have, for constants $C$ independent of $S$ and $R$,

$$
\begin{aligned}
\int_{\mathcal{O}_{S}}\left\{\frac{1}{2}\left|\nabla u_{R}\right|^{2}\right. & \left.+G\left(u_{R}\right)\right\} d x \leq \int_{\mathcal{O}_{S}}\left\{\frac{1}{2}\left|\nabla w_{R}\right|^{2}+G\left(w_{R}\right)\right\} d x \\
& =\int_{\mathcal{O}_{S-1}}\left\{\frac{1}{2}\left|\nabla w_{R}\right|^{2}+G\left(w_{R}\right)\right\} d x+\int_{\mathcal{O}_{S} \backslash \mathcal{O}_{S-1}}\left\{\frac{1}{2}\left|\nabla w_{R}\right|^{2}+G\left(w_{R}\right)\right\} d x \\
& \leq C\left|\mathcal{O}_{S-1} \cap\left\{\frac{s-t}{\sqrt{2}}<M\right\}\right|+C\left|\mathcal{O}_{S} \backslash \mathcal{O}_{S-1}\right| \\
& \leq C \int_{0}^{S-1}\left\{(t+\sqrt{2} M)^{m}-t^{m}\right\} t^{m-1} d t+C\left|B_{S} \backslash B_{S-1}\right| \\
& \leq C S^{2 m-1} .
\end{aligned}
$$

We have used the uniform gradient bound 3.6, the equality in $\mathcal{O}_{S-1}$ stated in 3.5 , and $G(M)=0$. We have also used the fact that $d x$ is equal to $c_{m} s^{m-1} t^{m-1} d s d t$ to 
bound the measure of the subset of $\mathcal{O}_{S-1}$, and that $(t+\sqrt{2} M)^{m}-t^{m} \leq C t^{m-1}$ and $S^{2 m}-(S-1)^{2 m} \leq C S^{2 m-1}$ for $t$ and $S$ larger than 1. We now let $R=R_{j} \rightarrow \infty$ to obtain

$$
\int_{\mathcal{O}_{S}}\left\{\frac{1}{2}|\nabla u|^{2}+G(u)\right\} d x \leq C S^{2 m-1}
$$

for some constant $C$ independent of $S$. Note that this bound, after odd reflection with respect to $\mathcal{C}$, establishes the energy bound

$$
\mathcal{E}\left(u, B_{S}\right) \leq C S^{2 m-1},
$$

which is estimate (1.15) in the statement of the theorem.

Suppose that $u \equiv 0$. Then the energy bound (3.7) would read

$$
c_{m} G(0) S^{2 m}=G(0)\left|B_{S}\right|=\mathcal{E}\left(0, B_{S}\right) \leq C S^{2 m-1} .
$$

This is a contradiction for $S$ large, and thus $u \neq \equiv 0$.

Finally, we establish the last statement of the theorem on stability under perturbations vanishing on the Simons cone. We assume hypothesis (1.14) on the concavity of $f$ in $(0, M)$. Since $f(0)=0$, concavity leads to $f^{\prime}(w) \leq f(w) / w$ for all $w \in(0, M)$. Hence

$$
-\Delta u=f(u) \geq f^{\prime}(u) u \quad \text { in } \mathcal{O} .
$$

That is, $u$ is a positive supersolution for the linearized operator $-\Delta-f^{\prime}(u)$ at $u$ in all of $\mathcal{O}$. By a simple argument (see the proof of Proposition 4.2 of [2]), the value of the quadratic form $Q_{u}(\xi)$ is nonnegative for all $\xi \in C^{1}$ with compact support in $\mathcal{O}$ (and not necessarily depending only on $s$ and $t$ ). By an approximation argument, the same holds for all $\xi \in C^{1}$ with compact support in $\overline{\mathcal{O}}$ and vanishing on $\partial \mathcal{O}=\mathcal{C}$. Finally, by odd symmetry with respect to $\mathcal{C}$, the same is true for all $C^{1}$ functions $\xi$ with compact support in $\mathbb{R}^{2 m}$ and vanishing on $\mathcal{C}$.

\section{Pointwise estimate for saddle solutions}

In this section we prove Proposition 1.5 using an important estimate of Modica [20] and two elementary lemmas. In [20] Modica proved the following pointwise gradient bound for global solutions of semilinear elliptic equations.

Theorem 4.1 (Modica [20]). Let $G \in C^{2}(\mathbb{R})$ be a nonnegative function and $u$ be a bounded solution of $\Delta u-G^{\prime}(u)=0$ in $\mathbb{R}^{n}$. Then

$$
\frac{|\nabla u|^{2}}{2} \leq G(u) \quad \text { in } \mathbb{R}^{n} .
$$

In addition, if $G\left(u\left(x_{0}\right)\right)=0$ for some $x_{0} \in \mathbb{R}^{n}$, then $u$ is constant. 
In [20] this bound was proved under the hypothesis $u \in C^{3}\left(\mathbb{R}^{n}\right)$. The result as stated above, which applies to all solutions-recall that every weak solution is $C^{2, \alpha}\left(\mathbb{R}^{n}\right)$ since $G \in C^{2}(\mathbb{R})$ - was established in [10].

Note that 1-D solutions - the functions $u_{b, c}$ defined in (1.7) - turn (4.1) into an equality (see Lemma 4.3]. In 1994, Caffarelli, Garofalo, and Segàla [10] extended the previous result of Modica to a wider family of equations which includes operators such as the $p$-Laplacian and the mean curvature operator for graphs. They also established that if equality holds in 4.1) at some point of $\mathbb{R}^{n}$, then $u$ must a 1-D solution.

The following are two auxiliary lemmas towards Proposition 1.5 The first one provides a formula for the distance to the cone $\mathcal{C}$. The second one concerns increasing solutions of 11.1 in $\mathbb{R}$.

Lemma 4.2. For every point $x \in \mathbb{R}^{2 m}$, the distance from $x$ to the Simons cone $\mathcal{C}=$ $\{s=t\}$ is given by

$$
\operatorname{dist}(x, \mathcal{C})=\frac{|s-t|}{\sqrt{2}} .
$$

This formula can be found, and also proven rigorously, using the method of Lagrange multipliers. Next we give an alternative simple proof of it.

Proof of Lemma 4.2. Let $x=\left(x^{1}, x^{2}\right) \in \mathbb{R}^{2 m} \backslash \mathcal{C}$ and $x_{0}=\left(x_{0}^{1}, x_{0}^{2}\right) \in \mathcal{C}$. Let $s=\left|x^{1}\right|$, $t=\left|x^{2}\right|$, and $s_{0}=t_{0}=\left|x_{0}^{1}\right|=\left|x_{0}^{2}\right|$. We have

$$
\begin{aligned}
\left|x-x_{0}\right|^{2}=\left|x^{1}-x_{0}^{1}\right|^{2}+\left|x^{2}-x_{0}^{2}\right|^{2} & =s^{2}+t^{2}+2 s_{0}^{2}-2 x^{1} \cdot x_{0}^{1}-2 x^{2} \cdot x_{0}^{2} \\
& \geq s^{2}+t^{2}+2 s_{0}^{2}-2(s+t) s_{0} \\
& =\frac{(s-t)^{2}}{2}+\frac{1}{2}\left((s+t)-2 s_{0}\right)^{2} \\
& \geq\left(\frac{s-t}{\sqrt{2}}\right)^{2} .
\end{aligned}
$$

Next, given $x \in \mathbb{R}^{2 m}$ we show that $x_{0} \in \mathcal{C}$ can be chosen so that the two inequalities above are in fact equalities. In case $s>0$ and $t>0$, choose $x_{0}=\left(\alpha x^{1}, \beta x^{2}\right)=$ $\left(\alpha x_{1}, \ldots, \alpha x_{m}, \beta x_{m+1}, \ldots, \beta x_{2 m}\right)$, where $\alpha$ and $\beta$ are given by $\alpha s=\beta t=(s+t) / 2$. If either $s$ or $t$ are zero, say $s>0$ and $t=0$, choose $x_{0}=\left(x^{1} / 2, x^{1} / 2\right)$.

The proof of the following lemma, which follows from integrating the ODE $\ddot{u}-G^{\prime}(u)$ $=0$, can be found in [5] - see also a sketch of the proof below, after the statement.

Lemma 4.3. Let $G \in C^{2}(\mathbb{R})$. There exists a bounded function $u_{0} \in C^{2}(\mathbb{R})$ satisfying

$$
\ddot{u}_{0}-G^{\prime}\left(u_{0}\right)=0 \quad \text { and } \quad \dot{u}_{0}>0 \quad \text { in } \mathbb{R}
$$

if and only if there exist two real numbers $m_{1}<m_{2}$ for which $G$ satisfies

$$
\begin{gathered}
G^{\prime}\left(m_{1}\right)=G^{\prime}\left(m_{2}\right)=0, \\
G>G\left(m_{1}\right)=G\left(m_{2}\right) \quad \text { in }\left(m_{1}, m_{2}\right) .
\end{gathered}
$$


In that case we have $m_{1}=\lim _{\tau \rightarrow-\infty} u_{0}(\tau)$ and $m_{2}=\lim _{\tau \rightarrow+\infty} u_{0}(\tau)$. Moreover, the solution $u_{0}=u_{0}(\tau)$ is unique up to translations of the independent variable $\tau$.

Adding a constant to $G$, assume that

$$
G\left(m_{1}\right)=G\left(m_{2}\right)=0 .
$$

Then

$$
\dot{u}_{0}^{2} / 2=G\left(u_{0}\right) \quad \text { in } \mathbb{R} .
$$

If in addition

$$
G^{\prime \prime}\left(m_{1}\right) \neq 0 \quad \text { and } \quad G^{\prime \prime}\left(m_{2}\right) \neq 0,
$$

then

$$
0<\dot{u}_{0}(\tau) \leq C e^{-c|\tau|} \quad \text { in } \mathbb{R}
$$

for some positive constants $C$ and $c$, and

$$
\int_{-\infty}^{+\infty}\left\{\frac{1}{2} \dot{u}_{0}(\tau)^{2}+G\left(u_{0}(\tau)\right)\right\} d \tau<+\infty .
$$

Given $G$ satisfying (4.2)-(4.4), to construct $u_{0}$ we simply choose any $m_{0} \in\left(m_{1}, m_{2}\right)$ and define

$$
\phi(\sigma)=\int_{m_{0}}^{\sigma} \frac{d w}{\sqrt{2(G(w))}} \quad \text { for } \sigma \in\left(m_{1}, m_{2}\right) .
$$

Then let $u_{0}:=\phi^{-1}$ be the inverse function of $\phi$. This formula is found by multiplying $\ddot{u}-G^{\prime}(u)=0$ by $\dot{u}$ and integrating the equation-which also gives the necessity of conditions (4.2) and (4.3) for existence. The above definition of $u_{0}$ leads automatically to (4.5).

Under the hypothesis $G^{\prime \prime}\left(m_{i}\right) \neq 0, G$ behaves like a quadratic function near each $m_{i}$. Using the expression above for $\phi$, this shows that $\phi$ blows up logarithmically at $m_{i}$, and thus its inverse function $u_{0}$ attains its limits $m_{i}$ at $\pm \infty$ exponentially. From this and identity (4.5), the exponential decay 4.7) for $\dot{u}_{0}$ follows, as well as 4.8).

Next we prove our pointwise bound.

Proof of Proposition 1.5. Let $u$ be a bounded solution of $-\Delta u=f(u)$ in $\mathbb{R}^{2 m}$ that vanishes on the Simons cone $\mathcal{C}=\{s=t\}$. We wish to show that

$$
|u(x)| \leq\left|u_{0}\left(\frac{s-t}{\sqrt{2}}\right)\right| \quad \text { in } \mathbb{R}^{2 m} .
$$

First we prove that $|u|<M$. Arguing by contradiction, assume that $|u| \geq M$ somewhere. Since $u(0)=0$, there exists a point $x_{0}$ such that $u\left(x_{0}\right)= \pm M$. Then, by Modica's gradient bound 4.1 we have $\left|\nabla u\left(x_{0}\right)\right|^{2} \leq 2 G\left(u\left(x_{0}\right)\right)=2 G( \pm M)=0$. Therefore $G\left(u\left(x_{0}\right)\right)=0$ and, by the second part of Theorem 4.1, $u$ is constant. Since $u=0$ on the Simons cone, we must have $u \equiv 0$. This contradicts the assumption $|u| \geq M>0$ somewhere. 
Next, since $|u|<M$, we may write

$$
u(x)=u_{0}(v(x))
$$

for some function $v: \mathbb{R}^{2 m} \rightarrow \mathbb{R}$, where $u_{0}$ is the 1-D solution whose existence is given by Lemma 4.3 , with $m_{1}=-M$ and $m_{2}=M$, and such that $u_{0}(0)=0$. Now, Modica's estimate 4.1] written in terms of $v$ becomes

$$
\frac{1}{2} \dot{u}_{0}^{2}(v)|\nabla v|^{2} \leq G\left(u_{0}(v)\right) \quad \text { in } \mathbb{R}^{2 m} .
$$

Since $\dot{u}_{0}^{2} / 2 \equiv G\left(u_{0}\right)$ by 4.5 , the expression above leads to

$$
|\nabla v| \leq 1 \quad \text { in } \mathbb{R}^{2 m}
$$

Finally, since $u=0$ on $\mathcal{C}$, we also have $v=0$ on $\mathcal{C}$. Given $x \in \mathbb{R}^{2 m}$, let $x_{0} \in \mathcal{C}$ be such that $\left|x-x_{0}\right|=\operatorname{dist}(x, \mathcal{C})$. Then

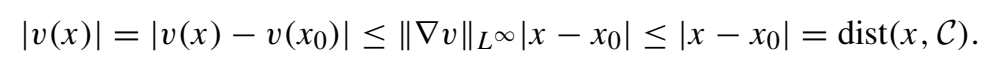

By Lemma 4.2, using the fact that $u_{0}$ is odd since $f$ is odd and that $u_{0}$ is increasing, we conclude

$$
|u(x)|=\left|u_{0}(v(x))\right|=u_{0}(|v(x)|) \leq u_{0}(\operatorname{dist}(x, \mathcal{C}))=\left|u_{0}\left(\frac{s-t}{\sqrt{2}}\right)\right|,
$$

which is the desired bound.

Finally, we prove the last statement of the proposition, that $u_{0}((s-t) / \sqrt{2})$ is a supersolution of $-\Delta u=f(u)$ in $\mathcal{O}=\{s>t\}$. First, direct computation using equation (1.17) in $(s, t)$ variables for $t>0$ shows that the function is a supersolution in $\{s>t>0\}$. In dimension $2 m \geq 4$ there is nothing else to be checked, by a capacity (or cut-off) argument used as in (3.3). Instead, in dimension $2, u_{0}((s-t) / \sqrt{2})$ is a supersolution in $\mathcal{O}$ since the outer flux $-\left.\partial_{t} u_{0}((s-t) / \sqrt{2})\right|_{t=0}=\dot{u}_{0}(s / \sqrt{2}) / \sqrt{2}$ is positive.

\section{Instability in dimension $n=4$}

In this section we prove the instability result of Theorem 1.4 For this, we establish that the function $u_{0}((s-t) / \sqrt{2})$ is unstable in dimension 4 in the sense that the second variation of energy $Q$ at $u_{0}((s-t) / \sqrt{2})$ is negative for some test function $\xi$ depending only on $s$ and $t$. Our proof also gives its instability outside every compact set. Even though $u_{0}((s-t) / \sqrt{2})$ is not a solution of the equation, we define the quadratic form

$$
Q_{u_{0}}(\xi):=\int_{\mathbb{R}^{2 m}}\left\{|\nabla \xi(x)|^{2}-f^{\prime}\left(u_{0}((s-t) / \sqrt{2})\right) \xi^{2}(x)\right\} d x,
$$

where there is some abuse of notation in writing $Q_{u_{0}}$ since by $u_{0}$ we really mean $u_{0}((s-t) / \sqrt{2})$. 
The key point of the proof is that $Q_{u_{0}}$ not being nonnegative definite leads to the same property for $Q_{u}$, where $u$ is any bounded solution that vanishes on the Simons cone. This fact will follow from our main pointwise bound of Proposition 1.5

For the proof it is useful to consider the variables

$$
y=\frac{s+t}{\sqrt{2}}, \quad z=\frac{s-t}{\sqrt{2}}
$$

which satisfy $-y \leq z \leq y$.

Recall that a bounded solution $u$ of $-\Delta u=f(u)$ in $\mathbb{R}^{2 m}$ is stable provided

$$
Q_{u}(\xi)=\int_{\mathbb{R}^{2 m}}\left\{|\nabla \xi|^{2}-f^{\prime}(u) \xi^{2}\right\} d x \geq 0 \quad \text { for all } \xi \in C_{c}^{\infty}\left(\mathbb{R}^{2 m}\right) .
$$

If $v$ is a function depending only on $s$ and $t$, the quadratic form $Q_{v}(\xi)$ acting on perturbations of the form $\xi=\xi(s, t)$ becomes

$$
c_{m} Q_{v}(\xi)=\int_{\{s>0, t>0\}} s^{m-1} t^{m-1}\left\{\xi_{s}^{2}+\xi_{t}^{2}-f^{\prime}(v) \xi^{2}\right\} d s d t,
$$

where $c_{m}>0$ is a constant depending only on $m$. We can further change to variables $(y, z)$ and obtain, for a different constant $c_{m}>0$,

$$
c_{m} Q_{v}(\xi)=\int_{\{-y<z<y\}}\left(y^{2}-z^{2}\right)^{m-1}\left\{\xi_{y}^{2}+\xi_{z}^{2}-f^{\prime}(v) \xi^{2}\right\} d y d z .
$$

Given the definition of the variables $y$ and $z$, a function $\xi=\xi(y, z)$ has compact support in $\mathbb{R}^{2 m}$ if and only if $\xi(y, z)$ vanishes for $y$ large enough.

Proof of Theorem 1.4 Let $u$ be a bounded solution of $-\Delta u=f(u)$ in $\mathbb{R}^{2 m}$ vanishing on the Simons cone $\mathcal{C}=\{s=t\}$. By Proposition 1.5. we know that

$$
|u(x)| \leq\left|u_{0}(z)\right| \quad \text { in all of } \mathbb{R}^{2 m} .
$$

This leads to $f^{\prime}(|u(x)|) \geq f^{\prime}\left(\left|u_{0}(z)\right|\right)$ for all $x \in \mathbb{R}^{2 m}$, since we assume $f$ to be concave in $(0, M)$. Now, since $f^{\prime}$ is even, we deduce that

$$
f^{\prime}(u(x)) \geq f^{\prime}\left(u_{0}(z)\right) \quad \text { for all } x \in \mathbb{R}^{2 m} .
$$

Therefore, since $Q_{u_{0}}$ is defined by (5.1) and $(s-t) / \sqrt{2}=z$, we conclude that

$$
Q_{u}(\xi) \leq Q_{u_{0}}(\xi) \quad \text { for all } \xi \in C_{c}^{\infty}\left(\mathbb{R}^{2 m}\right)
$$

It follows that, in order to prove that $u$ is unstable, it suffices to find a smooth function $\xi$ with compact support in $\mathbb{R}^{2 m}$ for which $Q_{u_{0}}(\xi)<0$. This is an easier task since $u_{0}(z)$ is explicit. Note also that, by an approximation argument, it suffices to find a Lipschitz function $\xi$, not necessarily smooth, with compact support in $\mathbb{R}^{2 m}$ and for which $Q_{u_{0}}(\xi)<0$. 
Expression (5.2) with $v(x)=u_{0}(z)$ reads

$$
c_{m} Q_{u_{0}}(\xi)=\int_{\{-y<z<y\}}\left(y^{2}-z^{2}\right)^{m-1}\left\{\xi_{y}^{2}+\xi_{z}^{2}-f^{\prime}\left(u_{0}(z)\right) \xi^{2}\right\} d y d z
$$

for all $\xi=\xi(y, z)$ with compact support in $\mathbb{R}^{2 m}$. We now take $\xi$ of separated variables, that is, of the form

$$
\xi(y, z)=\phi(y) \psi(z) .
$$

For $\xi$ to have compact support in $\mathbb{R}^{2 m}$ it suffices that $\phi$ has compact support in $y \in$ $(0,+\infty)$ (with no requirement on the support of $\psi$ ). Note also that $\xi$ is a Lipschitz function of $x \in \mathbb{R}^{2 m}$ if $\phi$ and $\psi$ are Lipschitz. However, even if $\phi$ and $\psi$ are smooth, $\xi$ will not be in general better than Lipschitz- to be smooth it would be necessary that the normal derivatives of $\xi$ vanish at $s=0$ and $t=0$ (i.e., at $z= \pm y$ ).

Since $\xi_{y}^{2}+\xi_{z}^{2}=\phi_{y}^{2} \psi^{2}+\phi^{2} \psi_{z}^{2}$, we have

$$
c_{m} Q_{u_{0}}(\xi)=\int_{\{-y<z<y\}}\left(y^{2}-z^{2}\right)^{m-1}\left\{\phi_{y}^{2} \psi^{2}+\phi^{2} \psi_{z}^{2}-f^{\prime}\left(u_{0}(z)\right) \phi^{2} \psi^{2}\right\} d y d z .
$$

Choose

$$
\psi(z)=\dot{u}_{0}(z) .
$$

We now let $2 m=4$ and thus $m-1=1$. In the following computations, we first integrate by parts the term $\left\{\left(y^{2}-z^{2}\right) \phi^{2} \psi_{z}\right\} \psi_{z}$ with respect to $z$ (note that here we obtain no boundary terms), and later we write the term $2 z \phi^{2} \psi \psi_{z}$ as $\phi^{2} z\left(\psi^{2}\right)_{z}$ and we integrate it by parts with respect to $z$. Thus,

$$
\begin{aligned}
& c_{m} Q_{u_{0}}(\xi)=\int_{\{-y<z<y\}}\left(y^{2}-z^{2}\right)\left\{\phi_{y}^{2} \psi^{2}+\phi^{2} \psi_{z}^{2}-f^{\prime}\left(u_{0}(z)\right) \phi^{2} \psi^{2}\right\} d y d z \\
&=\int_{\{-y<z<y\}}\left(y^{2}-z^{2}\right) \phi_{y}^{2} \psi^{2} d y d z+\int_{\{-y<z<y\}} 2 z \phi^{2} \psi \psi_{z} d y d z \\
& \quad-\int_{\{-y<z<y\}}\left(y^{2}-z^{2}\right) \phi^{2} \psi\left\{\psi_{z z}+f^{\prime}\left(u_{0}(z)\right) \psi\right\} d y d z \\
&=\int_{\{-y<z<y\}}\left(y^{2}-z^{2}\right) \phi_{y}^{2} \psi^{2} d y d z-\int_{\{-y<z<y\}} \phi^{2} \psi^{2} d y d z \\
&+\int_{0}^{+\infty} \phi^{2}(y)\left[z \psi^{2}(z)\right]_{-y}^{y} d y \\
&= \int_{\{-y<z<y\}}\left(y^{2}-z^{2}\right) \phi_{y}^{2} \psi^{2} d y d z-\int_{\{-y<z<y\}} \phi^{2} \psi^{2} d y d z+\int_{0}^{+\infty} \phi^{2}(y) 2 y \psi^{2}(y) d y \\
& \leq \int_{\{-y<z<y\}} y^{2} \phi_{y}^{2} \psi^{2} d y d z-\int_{\{-y<z<y\}} \phi^{2} \psi^{2} d y d z+\int_{0}^{+\infty} \phi^{2}(y) 2 y \psi^{2}(y) d y,
\end{aligned}
$$

where we have used the fact that $\psi=\dot{u}_{0}$ is an even function and a solution to the linearized 1-D problem $\psi_{z z}+f^{\prime}\left(u_{0}(z)\right) \psi=0$. 
For $a>1$ a constant that we will make tend to infinity, let $\eta=\eta(\rho)$ be a Lipschitz function of $\rho:=y / a$ with compact support $\left[\rho_{1}, \rho_{2}\right] \subset(0,+\infty)$. Let us denote by

$$
\phi(y)=\phi_{a}(y)=\eta(y / a) \quad \text { and } \quad \xi_{a}(y, z)=\phi_{a}(y) \dot{u}_{0}(z)=\eta(y / a) \dot{u}_{0}(z)
$$

the functions named $\phi$ and $\xi$ above. In the last bound for $Q_{u_{0}}$, we make the change $y=a \rho, d y=a d \rho$, and we use the fact that $\psi=\dot{u}_{0}$ is decreasing in $(0,+\infty)$. We obtain

$$
\begin{aligned}
& c_{m} Q_{u_{0}}\left(\xi_{a}\right) \\
& \leq \int_{\{-y<z<y\}} y^{2} \phi_{y}^{2} \psi^{2} d y d z-\int_{\{-y<z<y\}} \phi^{2} \psi^{2} d y d z+\int_{0}^{+\infty} \phi^{2}(y) 2 y \psi^{2}(y) d y \\
& \leq \int_{\{-a \rho<z<a \rho\}} a^{3} \rho^{2} \frac{\eta_{\rho}^{2}}{a^{2}} \psi^{2} d \rho d z-\int_{\{-a \rho<z<a \rho\}} a \eta^{2} \psi^{2} d \rho d z \\
&+\int_{\rho_{1}}^{\rho_{2}} a \eta^{2}(\rho) 2 a \rho \psi^{2}(a \rho) d \rho \\
&= a\left\{\int_{0}^{+\infty} \rho^{2}\left\{\eta_{\rho}^{2}-\frac{\eta^{2}}{\rho^{2}}\right\}\left\{\int_{-a \rho}^{a \rho} \dot{u}_{0}^{2} d z\right\} d \rho+2 a \rho_{2} \dot{u}_{0}^{2}\left(a \rho_{1}\right) \int_{0}^{+\infty} \eta^{2} d \rho\right\} .
\end{aligned}
$$

Dividing by $a$ leads to

$$
\frac{c_{m} Q_{u_{0}}\left(\xi_{a}\right)}{a} \leq \int_{0}^{+\infty} \rho^{2}\left\{\eta_{\rho}^{2}-\frac{\eta^{2}}{\rho^{2}}\right\}\left\{\int_{-a \rho}^{a \rho} \dot{u}_{0}^{2} d z\right\} d \rho+2 a \rho_{2} \dot{u}_{0}^{2}\left(a \rho_{1}\right) \int_{0}^{+\infty} \eta^{2} d \rho .
$$

Since $f$ is a concave function in $(0, M)$ with $f(0)=f(M)=0$, we have $f \geq 0$ in $(0, M)$. In addition, since $f \not \equiv 0$ in $(0, M)$ by $(1.13)$, we must have $f>0$ in $(0, M)$ by concavity. Now, $f$ being concave and positive in $(0, M)$ and with $f(M)=0$, we deduce that $f^{\prime}(M)<0$. Hence, $G^{\prime \prime}( \pm M)>0$, i.e., hypothesis 4.6 in Lemma 4.3 is satisfied. Thus by 4.7), we conclude that

$$
\lim _{a \rightarrow+\infty} a \dot{u}_{0}^{2}\left(a \rho_{1}\right)=0
$$

Therefore, letting $a \rightarrow+\infty$ in the last bound for $Q_{u_{0}}$ we obtain

$$
\limsup _{a \rightarrow+\infty} \frac{c_{m} Q_{u_{0}}\left(\xi_{a}\right)}{a} \leq\left\{\int_{-\infty}^{+\infty} \dot{u}_{0}^{2}(z) d z\right\} \int_{0}^{+\infty} \rho^{2}\left\{\eta_{\rho}^{2}-\frac{\eta^{2}}{\rho^{2}}\right\} d \rho .
$$

By 4.8 in Lemma $4.3, \dot{u}_{0}^{2}$ is integrable in $(-\infty,+\infty)$. Thus, by (5.3) and the comments after it, the proof of the instability of the solution $u$ will be finished if there exists a Lipschitz function $\eta=\eta(\rho)$ with compact support in $(0,+\infty)$ for which the second integral in (5.5) is negative.

Arguing by contradiction, assume that

$$
\int_{0}^{+\infty} \rho^{2} \frac{\eta^{2}}{\rho^{2}} d \rho \leq \int_{0}^{+\infty} \rho^{2} \eta_{\rho}^{2} d \rho
$$


for every Lipschitz function $\eta=\eta(\rho)$ with compact support in $(0,+\infty)$. The requirement that $\eta$ vanishes in a neighborhood of 0 can be removed by simply cutting-off $\eta$ in $(0, \varepsilon)$ and letting $\varepsilon \rightarrow 0$. The integrals in 5.6 can be seen as integrals in $\mathbb{R}^{3}$ of radial functions, that is, functions of the radius $\rho$. Hardy's inequality in $\mathbb{R}^{3}$ states that

$$
\frac{(3-2)^{2}}{4} \int_{\mathbb{R}^{3}} \frac{\eta^{2}}{|x|^{2}} d x \leq \int_{\mathbb{R}^{3}}|\nabla \eta|^{2} d x
$$

for every Lipschitz function $\eta$ with compact support in $\mathbb{R}^{3}$, and the constant $(3-2)^{2} / 4$ $=1 / 4$ is the best possible even when the inequality is considered only for radial functions (see [16]). Hence, since

$$
1>\frac{1}{4}=\frac{(3-2)^{2}}{4},
$$

5.6 leads to a contradiction, and this finishes the proof of instability.

The following is a direct way (without using Hardy's inequality) to see that the second integral in 5.5) is negative for some Lipschitz function $\eta$ with compact support in $(0,+\infty)$. For $\alpha>0$ and $0<2 \rho_{1}<1<\rho_{2}$, let

$$
\eta(\rho)= \begin{cases}\left(1-\rho_{2}^{-\alpha}\right) \rho_{1}^{-1}\left(\rho-\rho_{1}\right) & \text { for } \rho_{1} \leq \rho \leq 2 \rho_{1}, \\ 1-\rho_{2}^{-\alpha} & \text { for } 2 \rho_{1} \leq \rho \leq 1 \\ \rho^{-\alpha}-\rho_{2}^{-\alpha} & \text { for } 1 \leq \rho \leq \rho_{2} \\ 0 & \text { otherwise }\end{cases}
$$

a Lipschitz function with compact support $\left[\rho_{1}, \rho_{2}\right]$. We simply compute the second integral in 5.5 and find

$$
\begin{aligned}
& \int_{0}^{+\infty} \rho^{2}\left\{\eta_{\rho}^{2}-\frac{\eta^{2}}{\rho^{2}}\right\} d \rho \\
& \quad \leq \int_{\rho_{1}}^{2 \rho_{1}} \rho^{2}\left(1-\rho_{2}^{-\alpha}\right)^{2} \rho_{1}^{-2} d \rho+\int_{1}^{+\infty} \alpha^{2} \rho^{-2 \alpha} d \rho-\int_{1}^{\rho_{2}}\left(\rho^{-\alpha}-\rho_{2}^{-\alpha}\right)^{2} d \rho
\end{aligned}
$$

Choosing $1 / 2<\alpha<1$, as $\rho_{2} \rightarrow \infty$ the difference of the last two integrals converges to a negative number, since $\alpha^{2}<1$. Since the first of the last three integrals is bounded by $3 \rho_{1}$, we conclude that the above expression is negative by choosing $\rho_{2}$ large enough and then $\rho_{1}$ small enough.

The previous proof of instability also leads to the instability outside every compact set - and thus to the infinite Morse index property of $u$. Indeed, choosing $\rho_{1}$ and $\rho_{2}$ (and thus $\eta$ ) as above, we consider the corresponding function $\xi_{a}$ for $a>1$. Now, 5.3 and (5.5) lead to $Q_{u}\left(\xi_{a}\right) \leq Q_{u_{0}}\left(\xi_{a}\right)<0$ for $a$ large enough. Thus, the Lipschitz function $\xi_{a}$ makes the quantity $Q_{u}$ negative for $a$ large, and has compact support contained in $\left\{a \rho_{1} \leq(s+t) / \sqrt{2} \leq a \rho_{2}\right\}$. By approximation, the same is true for a function $\xi$ of class $C^{1}$, not only Lipschitz. Hence, given any compact set $K$ in $\mathbb{R}^{4}$, by taking $a$ large enough we conclude that $u$ is unstable outside $K$, as stated in the theorem. 
From the instability outside every compact set, it follows that $u$ has infinite Morse index in the sense of Definition 1.1 (c). Indeed, let $X_{k}$ be a subspace of $C_{c}^{1}\left(\mathbb{R}^{4}\right)$ of dimension $k$, generated by functions $\xi_{1}, \ldots, \xi_{k}$, and with $Q_{u}(\xi)<0$ for all $\xi \in X_{k} \backslash\{0\}$. Let $K$ be a compact set containing the support of all the functions $\xi_{1}, \ldots, \xi_{k}$. Since $u$ is unstable outside $K$, there is a $C^{1}$ function $\xi_{k+1}$ with compact support in $\mathbb{R}^{4} \backslash K$ for which $Q_{u}\left(\xi_{k+1}\right)<0$. Since $\xi_{k+1}$ has disjoint support with each of the functions $\xi_{1}, \ldots, \xi_{k}$, it follows that $\xi_{1}, \ldots, \xi_{k}, \xi_{k+1}$ are linearly independent and that $Q_{u}\left(a_{1} \xi_{1}+\right.$ $\left.\cdots+a_{k+1} \xi_{k+1}\right)=Q_{u}\left(a_{1} \xi_{1}+\cdots+a_{k} \xi_{k}\right)+Q_{u}\left(a_{k+1} \xi_{k+1}\right)<0$ for every nonzero linear combination $a_{1} \xi_{1}+\cdots+a_{k+1} \xi_{k+1}$ of them. We conclude that $u$ has infinite Morse index.

\section{Asymptotic stability of $u_{0}(z)$ in dimensions $2 m \geq 6$}

In this section we carry out, in all dimensions $n=2 m \geq 4$, the asymptotic analysis done for $n=4$ in the proof of Theorem 1.4 We will see that the argument does not lead to the instability of saddle solutions in dimensions $n=2 m \geq 6$. Indeed, we will show that $u_{0}(z)=u_{0}((s-t) / \sqrt{2})$ is, in every dimension $n=2 m \geq 6$ and only in some weak sense, asymptotically stable with respect to perturbations $\xi(y, z)$ with separated variables. Although this applies in dimension 6, note that $Q_{u} \leq Q_{u_{0}}$ is only an inequality, and thus $u_{0}$ may be asymptotically stable (or even stable) and at the same time the solution $u$ be unstable. Indeed, a more recent result of ours [9] establishes that saddle solutions in dimension 6 are unstable.

Recall that by (5.4), the second variation of energy at $u_{0}(z)$ applied to test functions $\xi(y, z)=\phi(y) \psi(z)$ has the form

$$
c_{m} Q_{u_{0}}(\xi)=\int_{\{-y<z<y\}}\left(y^{2}-z^{2}\right)^{m-1}\left\{\phi_{y}^{2} \psi^{2}+\phi^{2} \psi_{z}^{2}-f^{\prime}\left(u_{0}(z)\right) \phi^{2} \psi^{2}\right\} d y d z
$$

We choose, as in Theorem 1.4 $\psi(z)=\dot{u}_{0}(z)$-which is a solution of the linearized problem in $z$ and thus it should be the most unstable perturbation in the $z$ variable. We proceed as in the proof of Theorem 1.4 , integrating by parts the term $\left\{\left(y^{2}-z^{2}\right)^{m-1} \phi^{2} \psi_{z}\right\} \psi_{z}$ with respect to $z$, and later rewriting $\left(y^{2}-z^{2}\right)^{m-2} 2 z \phi^{2} \psi \psi_{z}$ as $\left(y^{2}-z^{2}\right)^{m-2} \phi^{2} z\left(\psi^{2}\right)_{z}$, and integrating it by parts with respect to $z$. Now there are no boundary terms when we integrate by parts since $m-2 \geq 1$. We obtain

$$
\begin{aligned}
c_{m} Q_{u_{0}}(\xi)= & \int_{\{-y<z<y\}}\left(y^{2}-z^{2}\right)^{m-1}\left\{\phi_{y}^{2} \psi^{2}+\phi^{2} \psi_{z}^{2}-f^{\prime}\left(u_{0}(z)\right) \phi^{2} \psi^{2}\right\} d y d z \\
= & \int_{\{-y<z<y\}}\left(y^{2}-z^{2}\right)^{m-1}\left\{\phi_{y}^{2} \psi^{2}-\phi^{2} \psi\left(\psi_{z z}+f^{\prime}\left(u_{0}(z)\right) \psi\right)\right\} d y d z \\
& +\int_{\{-y<z<y\}}(m-1)\left(y^{2}-z^{2}\right)^{m-2} 2 z \phi^{2} \psi \psi_{z} d y d z
\end{aligned}
$$




$$
\begin{aligned}
= & \int_{\{-y<z<y\}}\left(y^{2}-z^{2}\right)^{m-1} \psi^{2}\left\{\phi_{y}^{2}-\frac{m-1}{y^{2}-z^{2}} \phi^{2}\right\} d y d z \\
& +\int_{\{-y<z<y\}}(m-1)(m-2)\left(y^{2}-z^{2}\right)^{m-3} 2 z^{2} \phi^{2} \psi^{2} d y d z,
\end{aligned}
$$

where we have used the fact that $\psi=\dot{u}_{0}$ is a solution to the linearized 1-D problem $\psi_{z z}+f^{\prime}\left(u_{0}(z)\right) \psi=0$.

As before, let $a>1$ and $\phi(y)=\eta(y / a)$, where $\eta=\eta(\rho)$ is a function of $\rho=y / a$ with compact support in $(0,+\infty)$. Let

$$
\xi_{a}(y, z)=\xi(y, z)=\eta(y / a) \dot{u}_{0}(z) .
$$

Since $y=a \rho, d y=a d \rho$, we have

$$
\begin{aligned}
& c_{m} Q_{u_{0}}\left(\xi_{a}\right) \\
& =\int_{\{-a \rho \leq z \leq a \rho\}} a^{2 m-3} \rho^{2 m-2}\left(1-\frac{z^{2}}{a^{2} \rho^{2}}\right)^{m-1} \dot{u}_{0}^{2}\left\{\eta_{\rho}^{2}-\frac{m-1}{\rho^{2}\left(1-\frac{z^{2}}{a^{2} \rho^{2}}\right)} \eta^{2}\right\} d \rho d z \\
& \quad+\int_{\{-a \rho \leq z \leq a \rho\}} 2(m-1)(m-2) a^{2 m-5} \rho^{2 m-6} \eta^{2} \dot{u}_{0}^{2} z^{2}\left(1-\frac{z^{2}}{a^{2} \rho^{2}}\right)^{m-3} d \rho d z .
\end{aligned}
$$

Dividing by $a^{2 m-3}$ we deduce

$$
\begin{aligned}
\frac{c_{m} Q_{u_{0}}\left(\xi_{a}\right)}{a^{2 m-3}}= & \int_{\{-a \rho \leq z \leq a \rho\}} \rho^{2 m-2}\left(1-\frac{z^{2}}{a^{2} \rho^{2}}\right)^{m-1} \dot{u}_{0}^{2}\left\{\eta_{\rho}^{2}-\frac{m-1}{\rho^{2}\left(1-\frac{z^{2}}{a^{2} \rho^{2}}\right)} \eta^{2}\right\} d \rho d z \\
& +\int_{\{-a \rho \leq z \leq a \rho\}} \frac{2(m-1)(m-2)}{a^{2}} \rho^{2 m-6} \eta^{2} \dot{u}_{0}^{2} z^{2}\left(1-\frac{z^{2}}{a^{2} \rho^{2}}\right)^{m-3} d \rho d z .
\end{aligned}
$$

Since $0 \leq 1-z^{2} /\left(a^{2} \rho^{2}\right) \leq 1, m \geq 3$, and $z^{2} \dot{u}_{0}^{2}(z)$ is integrable in $(-\infty,+\infty)$, as $a \rightarrow \infty$ the second integral tends to zero and we are left with the expression

$$
\limsup _{a \rightarrow+\infty} \frac{c_{m} Q_{u_{0}}\left(\xi_{a}\right)}{a^{2 m-3}}=\left\{\int_{-\infty}^{+\infty} \dot{u}_{0}^{2} d z\right\} \int_{0}^{+\infty} \rho^{2 m-2}\left\{\eta_{\rho}^{2}-\frac{m-1}{\rho^{2}} \eta^{2}\right\} d \rho .
$$

According to Hardy's inequality for radial functions in $\mathbb{R}^{2 m-1}$ (see [16]), the last integral in $\rho$ is nonnegative for all $\eta=\eta(\rho)$ with compact support if and only if

$$
m-1 \leq \frac{(2 m-3)^{2}}{4} .
$$

When $2 m \geq 6$ this inequality is true (it is even strict) and we conclude some kind of asymptotic stability for $u_{0}(z)$.

Acknowledgments. The first author thanks Regis Monneau for interesting discussions and ideas on the subject of this paper.

Both authors were supported by MTM2005-07660-C02-01 and MTM2008-06349-C03-01. This work was part of the ESF Programme "GLOBAL".

The second author was supported by the FCT grant SFRH/BD/8985/2002. 


\section{References}

[1] Alama, S., Bronsard, L., Gui, C.: Stationary layered solutions in $\mathbb{R}^{2}$ for an Allen-Cahn system with multiple well potential. Calc. Var. Partial Differential Equations 5, 359-390 (1997) Zbl 0883.35036 MR 1450716

[2] Alberti, G., Ambrosio, L., Cabré, X.: On a long-standing conjecture of E. De Giorgi: symmetry in 3D for general nonlinearities and a local minimality property. Acta Appl. Math. 65, 9-33 (2001) Zbl 1121.35312 MR 1843784

[3] Alencar, H., Barros, A., Palmas, O., Reyes, J. G., Santos, W.: $\mathrm{O}(m) \times \mathrm{O}(n)$-invariant minimal hypersurfaces in $\mathbb{R}^{m+n}$. Ann. Global Anal. Geom. 27, 179-199 (2005) Zbl 1077.53007 MR 2131912

[4] Alessio, F., Calamai, A., Montecchiari, P.: Saddle-type solutions for a class of semilinear elliptic equations. Adv. Differential Equations 12, 361-380 (2007) Zbl pre05343690 MR 2305872

[5] Ambrosio, L., Cabré, X.: Entire solutions of semilinear elliptic equations in $\mathbb{R}^{3}$ and a conjecture of De Giorgi. J. Amer. Math. Soc. 13, 725-739 (2000) Zbl 0968.35041 MR 1775735

[6] Berestycki, H., Caffarelli, L., Nirenberg, L.: Further qualitative properties for elliptic equations in unbounded domains. Ann. Scuola Norm. Sup. Pisa Cl. Sci. 25, 69-94 (1997) Zbl 1079.35513 MR 1655510

[7] Bombieri, E., De Giorgi, E., Giusti, E.: Minimal cones and the Bernstein problem. Invent. Math. 7, 243-268 (1969) Zbl 0183.25901 MR 0250205

[8] Cabré, X., Capella, A.: On the stability of radial solutions of semilinear elliptic equations in all of $\mathbb{R}^{n}$. C. R. Math. Acad. Sci. Paris 338, 769-774 (2004) Zbl 1081.35029 MR 2059485

[9] Cabré, X., Terra, J.: Qualitative properties of saddle-shaped solutions to bistable diffusion equations. Forthcoming

[10] Caffarelli, L., Garofalo, N., Segàla, F.: A gradient bound for entire solutions of quasilinear equations and its consequences. Comm. Pure Appl. Math. 47, 1457-1473 (1994) Zbl 0819.35016 MR 1296785

[11] Dancer, E. N., Farina, A.: On the classification of solutions of $-\Delta u=e^{u}$ on $\mathbb{R}^{N}$ : stability outside a compact set and applications. Proc. Amer. Math. Soc. 137, 1333-1338 (2009) Zbl pre05544543 MR 2465656

[12] Dang, H., Fife, P. C., Peletier, L. A.: Saddle solutions of the bistable diffusion equation. Z. Angew. Math. Phys. 43, 984-998 (1992) Zbl 0764.35048 MR 1198672

[13] De Giorgi, E.: Convergence problems for functionals and operators. In: Proc. Int. Meeting on Recent Methods in Nonlinear Analysis (Rome, 1978), Pitagora, Bologna, 131-188 (1979). Zbl 0405.49001 MR 0533166

[14] Farina, A.: On the classification of solutions of the Lane-Emden equation on unbounded domains of $\mathbb{R}^{N}$. J. Math. Pures Appl. 87, 537-561 (2007) Zbl 1143.35041 MR 2322150

[15] Farina, A.: Stable solutions of $-\Delta u=e^{u}$ on $\mathbb{R}^{N}$. C. R. Math. Acad. Sci. Paris 345, 63-66 (2007) Zbl pre05182515 MR 2343553

[16] García Azorero, J. P., Peral, I.: Hardy inequalities and some critical elliptic and parabolic problems. J. Differential Equations 144, 441-476 (1998) Zbl 0918.35052 MR 1616905

[17] Ghoussoub, N., Gui, C.: On a conjecture of De Giorgi and some related problems. Math. Ann. 311, 481-491 (1998) Zbl 0918.35046 MR 1637919

[18] Giusti, E.: Minimal Surfaces and Functions of Bounded Variation. Birkhäuser, Basel-Boston (1984) Zbl 0545.49018 MR 0775682

[19] Jerison, D., Monneau, R.: Towards a counter-example to a conjecture of De Giorgi in high dimensions. Ann. Mat. Pura Appl. 183, 439-467 (2004) Zbl 1105.35037 MR 2140525 
[20] Modica, L.: A gradient bound and a Liouville theorem for nonlinear Poisson equations. Comm. Pure Appl. Math. 38, 679-684 (1985) Zbl 0612.35051 MR 0803255

[21] Modica, L.: Mortola, S.: Un esempio di $\Gamma^{-}$-convergenza. Boll. Un. Mat. Ital. B 14, 285-299 (1977) Zbl 0356.49008 MR 0445362

[22] Savin, O.: Regularity of flat level sets in phase transitions. Ann. of Math. 169, 41-78 (2009) MR 2480601

[23] Schatzman, M.: On the stability of the saddle solution of Allen-Cahn's equation. Proc. Roy. Soc. Edinburgh Sect. A 125, 1241-1275 (1995) MR 1363002

[24] Shi, J.: Saddle solutions of the balanced bistable diffusion equation. Comm. Pure Appl. Math. 55, 815-830 (2002) Zbl 1124.35316 MR 1894156

[25] Simons, J.: Minimal varieties in riemannian manifolds. Ann. of Math. 88, 62-105 (1968) Zbl 0241.53034 MR 0233295

[26] Villegas, S.: Asymptotic behavior of stable radial solutions of semilinear elliptic equations in $\mathbb{R}^{N}$. J. Math. Pures Appl. 88, 241-250 (2007) Zbl pre05200853 MR 2355457 